\title{
Quaderni
}

QUADERNI Communication, technologies, pouvoir

\author{
71 | Hiver 2009-2010 \\ Le changement climatique : les résistances à \\ l'adaptation
}

\section{Changement climatique et dépoldérisation : le rôle des acteurs et le poids des représentations sociales sur les côtes d'Europe atlantique}

\author{
Lydie Goeldner-Gianella
}

\section{OpenEdition \\ Journals}

Édition électronique

URL : http://journals.openedition.org/quaderni/527

DOI : 10.4000/quaderni.527

ISSN : 2105-2956

Éditeur

Les éditions de la Maison des sciences de l'Homme

Édition imprimée

Date de publication : 5 janvier 2010

Pagination : 41-60

\section{Référence électronique}

Lydie Goeldner-Gianella, " Changement climatique et dépoldérisation : le rôle des acteurs et le poids des représentations sociales sur les côtes d'Europe atlantique », Quaderni [En ligne], 71 | Hiver 2009-2010, mis en ligne le 05 janvier 2012, consulté le 23 avril 2019. URL : http:// journals.openedition.org/quaderni/527; DOI : 10.4000/quaderni.527 


\section{$D$ ossier}

\section{changement climatique et dépoldérisation :} le rôle des acteurs et le poids des représentations sociales sur les côtes d'Europe atlantique

\section{Lydie \\ Goeldner-Gianella}

Maître de Conférences

Université Paris 1

UMR 8586 PRODIG
Si la réalité du changement climatique et de ses impacts est désormais bien connue, rythmée par des conférences internationales régulières, beaucoup d'incertitudes demeurent, notamment dans les régions littorales, quant à l'ampleur de l'élévation du niveau marin ou la dangerosité des tempêtes. En Europe du Nord-Ouest, on s'attend à des problèmes d'érosion mais aussi de submersion marine des côtes endiguées, ce qui aura d'évidentes conséquences sociales et économiques. Pour y répondre, des techniques nouvelles d'adaptation au changement climatique telles que la « dépoldérisation », dont il sera question dans cet article, sont imaginées et testées par les acteurs de la défense contre la mer, mais selon des priorités et des modalités différentes selon les régions maritimes. On s'interrogera sur le rôle des acteurs publics dans ces politiques d'adaptation, mais aussi sur le rôle moins attendu mais néanmoins essentiel des acteurs associatifs et de la société civile locale, en Grande-Bretagne, aux Pays-Bas, en Allemagne et en France. Cet exemple illustrera l'importance d'une véritable « gestion intégrée de la zone côtière » dans un contexte de changement climatique, c'est-à-dire d'une gestion associant tous les acteurs à une échelle à laquelle c'est effectivement réalisable : l'échelle locale.

Dépoldériser dans un contexte de changement climatique

Incertitudes et impacts du changement climatique sur les côtes d'Europe atlantique

L'élévation du niveau de la mer a récemment pris de l'ampleur et devrait encore croître au $\mathrm{XXI}^{\mathrm{e}}$ siècle selon les prévisions du Groupe d'Experts 
Intergouvernemental sur l'Évolution du Climat (GIEC). Sur certaines côtes européennes, si l'on tient compte de variations régionales du niveau de la mer et des incertitudes relatives aux impacts de l'Oscillation Nord-Atlantique sur les niveaux marins hivernaux, l'on pourrait atteindre une fourchette d'élévation comprise entre une trentaine de centimètres et $1,50 \mathrm{~m}$ (Alcamo et al., 2007). La nouvelle Commission Delta envisage elle aussi pour les côtes néerlandaises une élévation pouvant aller jusqu'à $1,30 \mathrm{~m}$ en 2100 .

Cependant la relative précision de ces chiffres oblitère le fait qu'il ne s'agit que d'estimations basses ne prenant pas en considération la fonte des inlandsis, non complètement modélisée. Le GIEC concède qu'une élévation marine de plusieurs mètres pourrait se produire en l'espace de quelques siècles (GIEC, 2007). Mais certains chercheurs estiment qu'elle pourrait atteindre au moins 2 mètres dès le XXI ${ }^{\mathrm{e}}$ siècle, si les émissions de GES en restent à leur rythme actuel (Hansen et al., soumis). D'autres considèrent toutefois cette dernière estimation comme très improbable (Pfeffer et al., 2008), car elle supposerait que toutes les variables prises en considération atteignent rapidement des limites extrêmes. Malgré ces incertitudes, on estime que ce ne sont plus les estimations les plus basses du GIEC qui sont les plus justes. Ainsi, les estimations hautes envisagées ci-dessus pour l'Europe pourraient se révéler assez justes. En parallèle, le GIEC insiste sur un risque plus élevé de tempêtes hivernales en Europe atlantique, entraînant une augmentation de l'érosion comme des submersions. La Belgique, les Pays-Bas et l'Allemagne sont particulièrement menacés de submersion, avec $85 \%$ - pour les deux premiers - et $50 \%$ de leur littoral situés sous la cote des 5 mètres (EEA, 2005). Toutefois, de nombreuses incertitudes demeurent en ce qui concerne l'évolution de la tempétuosité (Tabeaud, 2007).

L'élévation eustatique étant une réalité, les pays d'Europe atlantique ont commencé à concevoir des formes d'adaptation au changement climatique. Les limites supérieures du niveau marin prises en compte dans ces politiques peuvent différer d'un pays à l'autre, en fonction de la précision des estimations régionales, mais aussi du degré de préoccupation à cet égard. Certains pays ont aussi intégré à leur réflexion l'idée d'un accroissement futur de la tempétuosité, à l'exception de la France où aucun accroissement n'a été remarqué dans les dernières décennies (ONERC, 2007).

Ainsi, la Grande-Bretagne base sa politique d'adaptation sur quatre scénarios, estimant une élévation du niveau marin globalement comprise entre 7 et $36 \mathrm{~cm}$ d'ici 2050 et une augmentation de la fréquence des tempêtes et des grandes vagues, de même que des niveaux d'eau atteints dans ces conditions (DEFRA, 2006). En France, l'étude lancée par le Conservatoire du littoral sur les côtes métropolitaines se fondait, en 2002, sur une estimation d'élévation de $44 \mathrm{~cm}$ d'ici 2100 et le rapport français à la dernière convention cadre des Nations Unis sur les changements climatiques (2006) évoquait une élévation de 30 à $50 \mathrm{~cm}$ (MEDD, 2006). Le rapport néerlandais évoquait pour sa part une élévation d'une soixantaine de centimètres d'ici 2100, et la commission Delta de $80 \mathrm{~cm}$. Plus au nord, le groupe de travail sur la mer des Wadden envisage une élévation séculaire d'une centaine de centimètres (CWSS, 2001) et 
une augmentation de la fréquence des tempêtes.

En termes démographiques, économiques et spatiaux, les impacts même incertains de ces modifications sont importants : Nicholls et Klein (EEA, 2005) ont établi que, dans le cas de submersions liées à une élévation de $1 \mathrm{~m}$ du niveau marin, les Pays-Bas et l'Allemagne verraient $67 \%$ et $4 \%$ de leurs populations respectives potentiellement affectées, de même que $69 \%$ et $30 \%$ de leur PIB ou $6,7 \%$ de leurs terres (Pays-Bas). Les littoraux de ces deux pays seraient ainsi les plus affectés en Europe par le changement climatique. Dans ces conditions, les coûts nécessaires à une adaptation seront particulièrement élevés. Il est toutefois démontré qu'une adaptation préventive serait moins coûteuse qu'une réaction tardive et au coup par coup : le livre vert de l'Europe sur l'adaptation au changement climatique estime notamment, dans l'hypothèse d'une élévation du niveau marin de $56 \mathrm{~cm}$ durant le $\mathrm{XXI}^{\mathrm{e}}$ siècle, que si des mesures d'adaptation étaient prises dès maintenant, les coûts annuels des dommages seraient trois fois moindres dans la décennie 2020 et six fois moindres dans la décennie 2080 (Commission des Communautés européennes, 29.6.2007). L'étude de Nicholls et Klein a montré que les coûts d'adaptation à une élévation marine d'un mètre correspondraient à 5,5\% et 2,2 \% des PIB respectifs des Pays-Bas et de l'Allemagne, ce qui resterait très inférieur au coût potentiel des dommages sans adaptation (EEA, 2005). D'autres modélisations réalisées sur l'ensemble de l'Europe parviennent à des conclusions similaires (EEA, 2007). Le temps d'agir est donc clairement venu ; c'est ce qui explique les choix déjà faits pour certains pays entre politiques offensives ou défensives face à la mer.

\section{Une solution face à l'élévation du niveau ma- rin : dépoldériser}

Certains États ont effectivement opté pour ce qu'on appelle depuis peu la dépoldérisation, comme nouvelle forme d'adaptation à l'élévation marine. Cette expression évoque simultanément plusieurs éléments : un milieu géographique tout d'abord - le polder -, un processus initial - la poldérisation - et sa fin - la dé-poldérisation, par conséquent -, mais aussi l'intervention humaine. En effet, dépoldériser, c'est renoncer à l'occupation et à l'exploitation humaines d'un polder - espace clos, conquis par l'homme sur la mer par un endiguement, puis asséché par le drainage à des fins agricoles. On parle de dépoldérisation partielle lorsque des ouvertures sur la mer ont été installées dans le corps d'une digue sous forme de tuyaux, de clapets, de portes à marée ou d'écluses. L'entrée des eaux est alors limitée, voire régulée. Pour dépoldériser complètement, on peut ouvrir une ou plusieurs brèches dans une digue, comme cela se produit accidentellement lors des tempêtes. Enfin, on peut opérer, mais c'est plus rare, un véritable démantèlement de la digue de mer, synonyme de complète disparition de cette digue et submersion d'un polder. Si la dépoldérisation continue de rester une forme minoritaire de gestion du littoral, c'est néanmoins un mouvement en plein essor depuis les années 1970, qui concerne déjà plus de $80 \mathrm{~km}^{2}$ et une centaine de polders en Europe occidentale. Ces dépoldérisations répondent à des objectifs de plus en plus variés et combinés, qui sont le fait d'une large palette d'acteurs publics et privés. Elles n'émanent plus uniquement, comme on le pense communément, de l'esprit de quelques écologistes soucieux de restaurer la 
nature, mais répondent à de véritables enjeux. En l'occurrence, un quart de ces dépoldérisations est à vocation défensive, c'est-à-dire en rapport avec l'élévation du niveau de la mer. Si nos propos se centrent dans cet article sur ce type particulier de dépoldérisation, il en existe d'autres types, à visée environnementale, à visée économique ou à visée globale (Goeldner-Gianella, 2008).

Ces dépoldérisations défensives constituent une des formes d'adaptation au changement climatique. Sur les côtes endiguées, on peut classiquement résister à la mer en rehaussant les digues mais ce n'est pas une technique durable, car elles atteignent déjà par endroits près d'une dizaine de mètres - ou en continuant d'avancer sur elle, par endiguement ou remblaiement. Toutefois, la protection grandissante des zones humides depuis les années 1970 entrave fortement le recours à la poldérisation car celle-ci s'opère aux dépens de milieux écologiquement très riches (vasières et prés salés). Il en est tout autrement de la technique de la dépoldérisation, qui permet justement de se défendre contre la mer sans dommages environnementaux. Cette technique permet en effet de reconstituer des milieux naturels : en reculant la digue et en permettant à la mer de pénétrer dans un polder, à travers une ou plusieurs brèches, un processus de sédimentation entraîne une renaissance relativement rapide des vasières et des prés salés, qui peuvent alors aider à freiner la houle. Appelée « managed realignment » en GrandeBretagne, c'est-à-dire réalignement contrôlé (d'une digue), la dépoldérisation permet d'augmenter l'efficacité et la pérennité des digues. En effet, lorsqu'un pré salé est suffisamment large, il peut jouer le rôle d'un espace tampon où la houle, freinée, s'atténue progressivement, ce qui permet de ne plus craindre de niveaux d'eau trop élevés et de ne pas engager de politique trop coûteuse de surélévation systématique des digues. Les ingénieurs britanniques ont établi, dans les années 1990, des rapports précis entre largeur du schorre, hauteur des digues et coût de construction par mètre linéaire (NRA, s.d.n.1.). Cette politique de managed realignment a été largement adoptée sur les côtes endiguées de Grande-Bretagne (DEFRA et al., 2002), nombreuses sur la face sud-est du pays donnant sur la mer du Nord, où l'élévation eustatique est accentuée par une subsidence glacio-isostatique.

\section{Les particularités des "dépoldérisations défensives "}

Ces dépoldérisations défensives présentent la particularité d'être concentrées en quelques secteurs en Europe. En l'occurrence, 70 \% d'entre elles se localisent sur les côtes britanniques en 2008, le reste sur les côtes belges et néerlandaises, particulièrement dans la partie méridionale de la mer du Nord. C'est la survenue régulière de tempêtes dans cette région maritime qui est en partie à l'origine de cette politique. Les lames de tempête sont fréquentes dans le nord-ouest de l'Europe sur le passage des dépressions de la zone tempérée. Elles s'avèrent particulièrement dangereuses dans la partie sud de la mer du Nord, plus resserrée, où les vents venant du nord-ouest peuvent pousser les eaux vers les côtes de l'Angleterre orientale, des Pays-Bas et de la Belgique. Les surcotes ainsi créées par rapport au niveau attendu de la marée sont particulièrement dangereuses si elles se conjuguent à une marée de vive-eau, comme ce fut le cas en février 1953 aux Pays-Bas et an Angleterre. Or, ces lames de tempête frappent ré- 
gulièrement ces côtes du fait de la forte fréquence des tempêtes dans l'Atlantique Nord (une par jour en moyenne en hiver) et de leur emprunt régulier du même rail, grossièrement situé entre les $50^{\mathrm{e}}$ et le $60^{\mathrm{e}}$ parallèles dans le secteur de la mer du Nord (Schoenenwald, 2003).

En réponse à ces tempêtes, outre la dépoldérisation, il existe dans les estuaires d'autres techniques d'adaptation à la montée des eaux. Il s'agit de dépoldérisations partielles qui fonctionnent sous la forme de polders de décharge ou de ce qu'on appelle en Belgique des « zones de régulation des inondations à marée réduite contrôlée ». Dans les polders sélectionnés à cette fin, les digues sont maintenues, mais arasées et équipés d'écluses, de façon à permettre une entrée par débordement des eaux estuariennes, en cas de tempête ou de marée exceptionnelle, et une sortie contrôlée de ces eaux à l'issue de la tempête. Cette rétention ponctuelle des eaux permet une diminution de la hauteur de la marée en amont du polder et, par conséquent, une réduction du risque de submersion. On s'est interrogé dans l'estuaire de l'Elbe, sur l'intérêt d'aménager de tels polders juste en aval de Hambourg, de façon à mieux protéger la ville contre la mer en l'absence d'un barrage anti-tempête. Cette option a été appliquée dans d'autres estuaires, tel l'Escaut maritime (partie de l'Escaut située entre Anvers et Gand), où la Belgique utilise les 580 ha du polder KBR pour réduire de cinq fois la probabilité d'une submersion du bassin versant. Dans la Humber, à Alkborough, une dépoldérisation par brèche récemment opérée dans un polder agricole de 440 ha doit permettre une réduction de $15 \mathrm{~cm} \mathrm{du}$ niveau des eaux lors des plus fortes marées, sur des dizaines de kilomètres à l'amont du site, le long de plusieurs affluents.

On notera avec intérêt que ce ne sont pas uniquement les acteurs publics de la protection contre la mer, en charge de la construction ou de l'entretien des digues, qui interviennent dans ces mesures d'adaptation, mais aussi les acteurs associatifs ou privés de la protection de la nature.

\section{Le rôle des acteurs de la protection contre la mer ou de la protection de la nature dans les dépoldérisations défensives}

Cette analyse du rôle des acteurs dans les dépoldérisations liées au changement climatique suppose des précisions liminaires. Ces mesures se subdivisent en effet, en 2008, en $50 \%$ de dépoldérisations à visée strictement défensive (ou défensive et environnementale) et $50 \%$ de dépoldérisations à visée défensive et compensatoire (figure 1). C'est cette complexité d'objectifs qui explique la variété des acteurs intervenant dans cette politique.

\section{Des dépoldérisations défensives clairement promues par les acteurs britanniques de la protection côtière}

Les dépoldérisations défensives strictes caractérisent tout particulièrement la GrandeBretagne où le retour de la mer a été institué en politique d'adaptation - bien qu'il ne s'agisse pas de la seule technique possible et utilisée. Le recours régulier au managed realignment s'explique par le fait que les digues sont en mauvais état et que le schorre (c'est-à-dire les prés salés) y a subi une forte réduction. Des 
mesures précises effectuées durant vingt-cinq ans (1973-98) le long des côtes de l'Essex montrent que le schorre ne s'étend plus que sur un quart de sa surface initiale (ce qui correspond à une perte moyenne de $40 \mathrm{ha} / \mathrm{an}$ ) (Cooper et al., 2001 / cf. photo 1). Les Britanniques craignent particulièrement le phénomène de "coastal squeeze », c'est-à-dire de compression des écosystèmes littoraux qui seraient contraints de migrer vers l'amont de l'estran, du fait d'une élévation eustatique, mais ne le pourraient pas en présence de structures défensives lourdes comme les digues. Dans l'estuaire de la Humber, des études prévisionnelles attribuent à cette compression littorale $85 \%$ des futures pertes d'habitats intertidaux (Edwards \& Winn,
2006). Or, le schorre a une fonction défensive essentielle en Grande-Bretagne, où il aide à protéger de l'érosion directe $2000 \mathrm{~km}$ de rivage - où sont d'ailleurs présents $1 \%$ de la population britannique et la moitié des meilleures terres agricoles du pays.

À l'amont du schorre, les digues et le barrage anti-tempêtes de la Tamise jouent aussi un rôle fondamental, protégeant 1,5 million de personnes et 160000 propriétés en Angleterre et aux Pays-de-Galles (Environment Agency, 1999). Or ces structures sont actuellement en mauvais état : $41 \%$ des structures de défense (dont les digues font partie) nécessitent des travaux de conservation modérés à significatifs et $33 \%$

Figure 1 : Types et répartition des dépoldérisations à visée défensive, réalisées ou projetées sur les côtes européennes

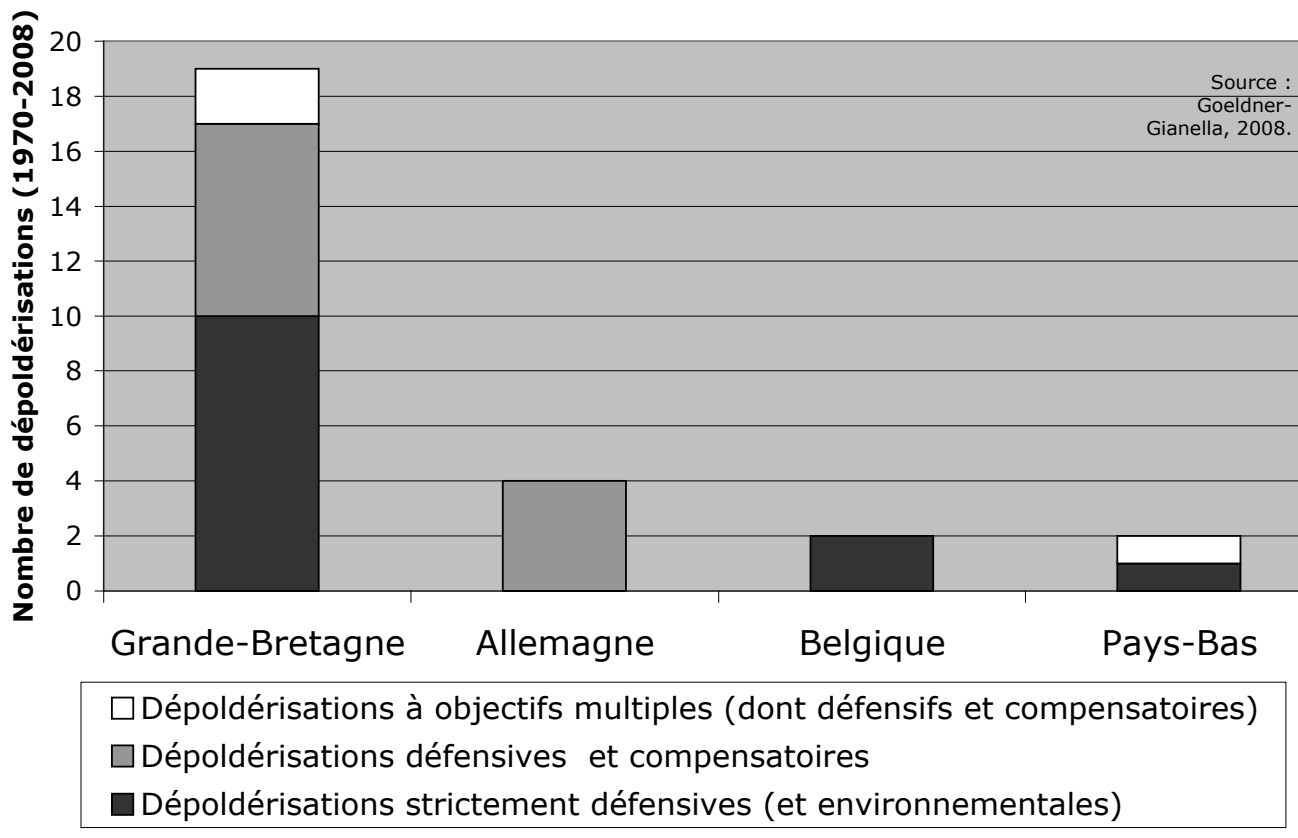




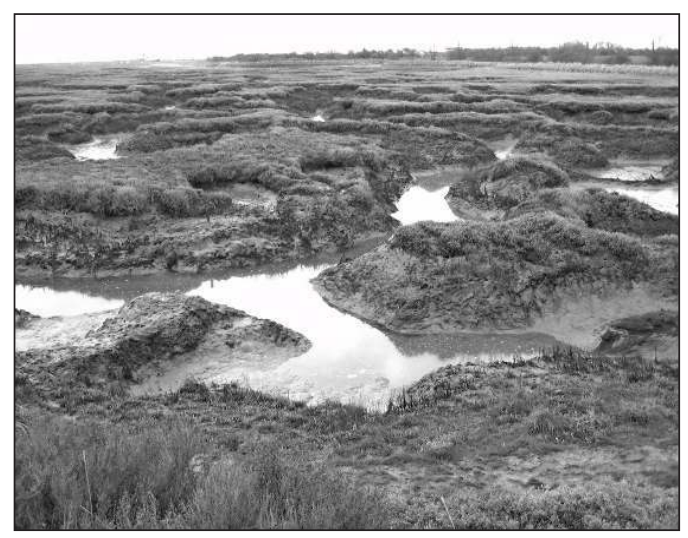

Photo 1 : la forte érosion du schorre de Tollesbury (Blackwater estuary, Essex) a justifié une dépoldérisation défensive (mars 2008).

avaient, en 1999, une durée de vie inférieure à dix ans (Rupp-Armstrong \& Nicholls, 2007)! D'après des études prospectives, les dégâts associés aux tempêtes et aux submersions pourraient être deux à trois fois plus élevés qu'actuellement et l'investissement nécessaire au renforcement et au maintien des structures défensives, en Angleterre et au Pays-de-Galles, pourrait atteindre en moyenne 50 millions de livres par an (Department of Environment, Transport and Regions, 2000). Le choix de réaligner les digues plutôt que de les renforcer s'impose donc naturellement.

Le gouvernement britannique révise actuellement sa stratégie de protection contre la mer. En 2000, le ministère de l'Agriculture a lancé une étude sur le devenir des sites littoraux Natura 2000 (Lee, 2001). En 2004, le ministère de l'Agriculture a estimé que $5000 \mathrm{~km}^{2}$ de terres agricoles étaient des « candidats potentiels pour des politiques de restauration et de réalignement fluvial et côtier » (Rupp-Armstrong \& Nicholls, 2007). Et en 2005, le gouvernement a publié sa nouvelle stratégie :
Making space for water (DEFRA, 2005). L'intérêt du managed realignment, le long des côtes comme le long des rivières, y est clairement souligné. Les espaces ruraux serviront en particulier à limiter les risques car on y donnera plus de place à l'eau, en recréant des zones humides, en opérant des retraits de digues, en créant des zones inondables - tels les polders de décharge. De plus, l'aide publique au financement des défenses côtières ne se poursuivra que lorsque ces défenses induiront de multiples bénéfices justifiant les dépenses. La Grande-Bretagne est la seule à avoir prévu d'appliquer une politique de retrait stratégique des digues à une si large échelle.

\section{L'essor de dépoldérisations défensives et com- pensatoires promues par des acteurs variés}

Dans la moitié des cas, ces dépoldérisations à visée défensive se doublent d'un objectif de compensation, lié à l'obligation de répondre aux dommages causés à l'environnement littoral par de grands travaux. Ces travaux relèvent soit de la politique de protection côtière - endiguements défensifs encore réalisés dans les années 1980/90 ou récents rehaussements de digues du fait du changement climatique -, soit de la politique de développement industriel et portuaire - remblaiements pour agrandir les zones d'accueil des conteneurs. Ce type de dépoldérisation se localise préférentiellement dans le nord-ouest de l'Europe, où sévissent les tempêtes de la mer du Nord et se concentrent les ports de la Northern Range et leurs projets répétés d'extension.

Par exemple, dans l'estuaire de l'Elbe, les dépoldérisations mises en place dans les années 1990 à Hambourg relèvent de mesures 
compensatoires en rapport avec des élargissements de digues. Il est assez paradoxal de noter qu'on ouvre des digues pour recréer des zones humides car leur élargissement, quelques centaines de mètres plus loin, a fait reculer ces mêmes zones humides... Des compensations de travaux portuaires par des mesures de dépoldérisation ont été mises en œuvre en Allemagne, aux PaysBas et en Grande-Bretagne, notamment dans les estuaires de grand trafic portuaire : Elbe, Weser, Escaut occidental, Tamise, Humber. Ainsi dans la Humber, le groupe privé British Ports Associated a dû compenser des remblaiements de vasières dans les ports de Hull et d'Immingham par les dépoldérisations de Chowder Ness et de Welwick. En parallèle et officiellement, ces dépoldérisations compensatoires favorisent la politique publique de défense côtière.

\section{Des dépoldérisations défensives et environne- mentales promues par les acteurs de la protec- tion de l'environnement}

Il convient également de parler de dépoldérisations défensives et environnementales, qui sont notamment mises en œuvre en Grande-Bretagne. La reconstitution de zones humides - vasières et schorres - n'a évidemment pas pour seul but l'adaptation au changement climatique. Il s'agit aussi de faire renaître des écosystèmes qui ont largement disparu sous l'effet des aménagements anthropiques. Au cours des deux derniers siècles, les estuaires britanniques ont ainsi perdu par endiguement 25 à $50 \%$ de leur zone intertidale et la moitié de leurs schorres, alors qu'il s'agit de milieux à forte biodiversité et productivité. Dès lors, les dépoldérisations défensives britanniques sont systématiquement à comprendre comme des dépoldérisations qui ont simultanément une visée environnementale, si bien que les protecteurs de la nature se sont, eux aussi, emparés de cette technique. Ainsi, la RSPB britannique (Royal Society for the Protection of Birds), dont on connaît la notoriété séculaire et le grand nombre d'adhérents, a indirectement contribué à une dépoldérisation défensive et environnementale à Freiston Shore, dans le Wash, et directement entrepris trois d'entre elles : celle de Nigg Bay dans le Cromarty Firth en 2003 et celles de Wallasea Island (Crouch, Essex) et de Skinflats (Forth, Ecosse), encore à l'état de projets. L'association Essex Wildlife Trust a, elle aussi, participé à la dépoldérisation défensive et environnementale d'Abbotts Hall Farm en 2002 (Essex). Il est vrai que le statut de la propriété sur l'estran facilite grandement ces interventions en Grande-Bretagne, les associations pouvant être propriétaires des digues qui ceinturent leurs polders, mais aussi des marais maritimes adjacents ou recréés par la dépoldérisation, - alors qu'ailleurs en Europe, ce statut peut interdire des interventions directes sur les digues ou sur l'estran, voire imposer la propriété publique de l'estran (Goeldner-Gianella, 2008).

Si les dépoldérisations menées par des associations ou des $\mathrm{ONG}^{1}$ n'ont pas toutes des objectifs défensifs directs, on observe qu'une alliance d'objectifs entre la défense contre la mer et la restauration écologique intéresse de plus en plus les acteurs de la protection de l'environnement, qui y voient peut-être une forme d'intervention socialement plus acceptable. En Allemagne notamment, de grandes associations ont été associées aux projets de dépoldérisation menées dans les années 1980/90 par les ingénieurs de la 
défense côtière dans le Schleswig-Holstein. Par ailleurs, le WWF allemand s'est très tôt intéressé au thème de la dépoldérisation (Marchand \& Nolte, 1995). Et actuellement, la section WWF du Schleswig-Holstein travaille à une évolution des mentalités des ingénieurs allemands, pour promouvoir l'acceptation de techniques plus durables de défense contre la mer, telles que la dépoldérisation. Pour se forger des arguments, cette section a visité en 2008 les sites dépoldérisés de la RSPB !

En France, si les dépoldérisations du Conservatoire du Littoral ne sont pas à visée défensive directe, elles ont toutefois un rapport étroit avec l'élévation du niveau de la mer. Les chercheurs estiment que les côtes à marais maritimes et les digues souffriront globalement peu, en France, de l'érosion liée au changement climatique (Paskoff, 2004 ; www.eurosion.org), mais l'augmentation des tempêtes conduira à des submersions plus fréquentes des polders de première ligne, avec ou sans brèche. En l'occurrence, de telles submersions se produisent déjà lors des plus fortes tempêtes : en décembre 1999, les côtes de la Charente et de la Gironde ont été inondées sur 12000 ha. Le Conservatoire du Littoral, qui est sans doute le plus grand propriétaire ${ }^{2}$ de polders de première ligne en France réfléchit par conséquent à leur devenir. La probabilité de submersion d'un polder dépend du fonctionnement de ses digues et donc de la politique de gestion des digues adoptée par l'établissement. Cette politique demande à être révisée par le Conservatoire dont 3300 ha, soit plus de $80 \%$ de ses polders, sont déjà exposés à un risque de submersion - cela représente $6,5 \%$ du patrimoine actuel de l'organisme (Clus-Auby et al., 2005). Ces résultats inciteront certainement cet organisme à rendre ces sites sensibles à la mer - ce qu'il a d'ailleurs déjà réalisé à plusieurs reprises, en laissant ouvertes des brèches qui s'étaient accidentellement produites. Il est vrai que la philosophie du Conservatoire est celle d'une absence de résistance à la mer, de façon à conserver les aspects naturels des sites et leurs équilibres écologiques. Ainsi, si l'objectif du Conservatoire n'est pas prioritairement défensif, comme dans le cas du managed realignment, il peut toutefois l'être indirectement, puisqu'il peut souhaiter - en acceptant des dépoldérisations accidentelles ou en dépoldérisant volontairementréduire le coût d'entretien de digues défectueuses, tout en contribuant à une restauration écologique (cf. photo 2).

Photo 2 : forte érosion de la digue frontale de Malprat, dans le bassin d'Arcachon (sept. 2008). On reconnaît les anciens piquets de fondation de la digue. Son recul actuel est clairement matérialisé par des piquets plus récents (à gauche des premiers), qui devaient aider pendant un temps à son renforcement. Le CEL a conservé les brêches qui se sont produites, acceptant cette dépoldérisation accidentelle pour réduire entre autres les coûts d'entretien des digues.

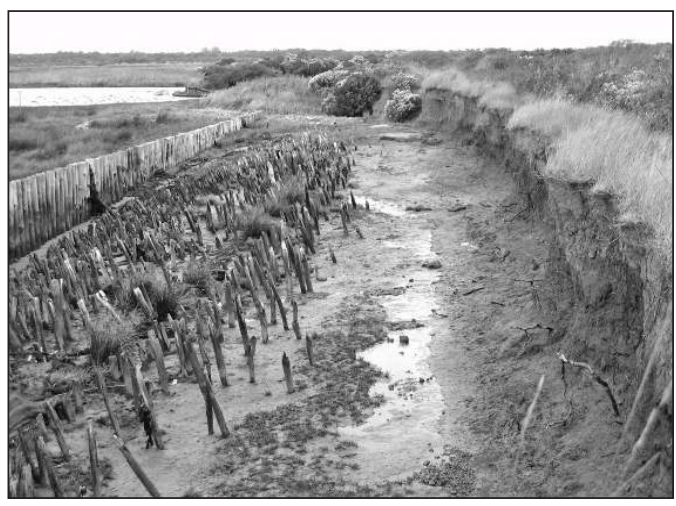


Le poids des représentations sociales et de la participation locale dans l'acceptation des dépoldérisations défensives

Si le rôle des maîtres d'ouvrage est fondamental dans l'adaptation au changement climatique, c'est aussi le cas des ingénieurs ou des scientifiques qui impulsent le recours à de nouvelles techniques de défense - ou des populations locales qui ont le pouvoir de freiner ou d'accélérer les choix proposés. Un travail de recherches en cours sur le rôle des ingénieurs dans la gestion du littoral (Guében-Venière, 2009) a par exemple montré que les ingénieurs français s'interrogeaient beaucoup sur l'évolution des tempêtes au XXI' siècle, mais sans avoir de position claire à l'égard de la dépoldérisation ou de connaissances précises sur le managed realignment. Ces conclusions ne sont certes pas généralisables, mais mettent en exergue l'importance d'une prise en compte des avis et des connaissances de la société civile, comme des groupes d'intérêts ou des groupes professionnels qui la composent (ingénieurs, écologistes etc.).

\section{L'influence des représentations sociales de la tempête sur la politique de dépoldérisation}

On observe, par exemple, que les tempêtes peuvent avoir une importance culturelle forte pour certaines sociétés littorales, importance ayant des implications sur le recours à la dépoldérisation. $\mathrm{Au} \mathrm{XX}^{\mathrm{e}}$ siècle, les tempêtes les plus dévastatrices de la mer du Nord ont été celles du 31 janvier au 2 février 1953, aux PaysBas et en Angleterre, et celle de février 1962 en Allemagne : du fait d'innombrables ruptures de digues, les victimes se sont comptées en centaines en Angleterre et en Allemagne et on a dénombré
1850 victimes aux Pays-Bas, notamment en Zélande. Lors de tempêtes ultérieures, le niveau marin a parfois atteint celui de 1953 - en janvier 1976 et novembre 2007 dans le sud-ouest des Pays-Bas - mais des mesures de protection côtière prises à partir des années 1950/60 ont permis d'y résister (plan Delta aux Pays-Bas, plans généraux de protection côtière en Allemagne, plan Sigma en Belgique, barrage de la Tamise en Angleterre).

Une comparaison de la situation des trois pays montre que les Pays-Bas ont été les plus durement touchés au XX ${ }^{\mathrm{e}}$ siècle. La Zélande a connu en 1953 une véritable « catastrophe nationale », comptant beaucoup plus de victimes, de surfaces inondées, d'animaux noyés et de bâtiments détruits que ses voisins britanniques (en 1953) et allemand (en $1962)^{3}$. Le fort impact psychologique de cette tempête pour les Néerlandais s'explique aussi par sa très forte concentration géographique dans pratiquement une seule région (la Zélande et le sud de la Hollande méridionale), alors que les zones submergées étaient davantage disséminées sur la côte anglaise, du Lincolnshire au Kent. La pénétration des eaux dans l'arrièrepays a également davantage frappé les Pays-Bas que l'Angleterre, de surcroît dans des régions agricoles aux densités plus élevées (GoeldnerGianella, 2008).

Les tempêtes de 1953 et 1962 ont ainsi eu des effets psychologiques contrastés. Rupp-Armstrong et Nicholls (2007) considèrent que la tempête de 1953 a été oubliée des Britanniques, alors que sur la côte de la mer des Wadden, les Allemands se remémorent encore celle de 1962. Cette indifférence britannique est confirmée par 
les enquêtes de Myatt, Scrimshaw et Lester (2003 $\mathrm{a}, \mathrm{b}, \mathrm{c})$, dans les secteurs dépoldérisés des côtes du North Norfolk et de l'Essex. Elles montrent une absence systématique de crainte à l'égard de futures submersions : la population paraît, tout au plus, modérément inquiète. Les chercheurs observent aussi une grande confiance de la société dans le système défensif et insistent sur l'idée que « les tempêtes du passé sont un événement inhabituel, peu susceptible de se reproduire » (une assertion qui regroupe environ un tiers des réponses à Freiston Shore et la moitié à Orplands). Les opérateurs de dépoldérisations que nous avons interrogés sur le managed realignment adhèrent eux aussi à l'idée que la tempête de 1953 est sortie des mémoires et que la population n'est préoccupée que par les conséquences financières des submersions et plus particulièrement des inondations continentales. La confiance dans le bon état des digues maritimes (!) et dans le système d'alerte littoral est telle que personne ne songe à un risque vital... Aux Pays-Bas a contrario, le drame de 1953 a considérablement marqué les esprits, persistant jusqu'à nos jours dans les mémoires du fait de sa survenue à une époque où les digues auraient dû permettre de résister à la mer. La fragilité de l'homme et du pays tout entier a été violemment remise en évidence à cette occasion, malgré la tradition de courage et de combativité de la Zélande dont la devise face à la mer a toujours été «luctor et emergo » ${ }^{4}$. Comme le dit P. Leroy (2006), « les Pays-Bas ne peuvent se comprendre sans cette relation amour-haine existentielle, il n'y a pas d'autre mot, avec l'eau ». A. Miossec (1993) parle d'un pays " au péril de la mer», " où la fragilité est d'abord celle de la côte face à l'océan déchaîné : les souvenirs cruels de 1953 pèseront longtemps sur le comportement des populations. Tenir face aux flots, voici la règle cardinale d'une bonne gestion du littoral ». Par conséquent, de peur que l'oubli ne se développe parmi les jeunes générations, des panneaux ont été implantés pour rappeler quelle serait la position naturelle de la ligne de rivage si des polders n'avaient pas été construits sur plus d'un tiers du pays (Leroy, 2006). Sur l'île zélandaise de Schouwen-Duiveland, presque complètement submergée en 1953, un musée commémoratif du désastre a récemment ouvert.

Dans ce contexte, on comprend que les Zélandais réprouvent l'idée de dépoldérisation : les polders ont été difficilement conquis ; ils font la fierté du pays; les rendre à la mer serait un " gâchis ». Cette fierté nationale est inculquée dès l'école primaire et même les écologistes s'y disent sensibles (V. Klap, ZMF, comm. pers.). Pour les personnes plus âgées qui ont vécu la tempête de 1953, l'émotion entre aussi en considération dans ce refus, car les plaies sont restées vives. Les Zélandais frappés personnellement en 1953 ou concernés

Photo 3 : monument commémoratif de la tempête de février 1953 en Zélande. La faiblesse de l'homme face aux flots marins est clairement exprimée.

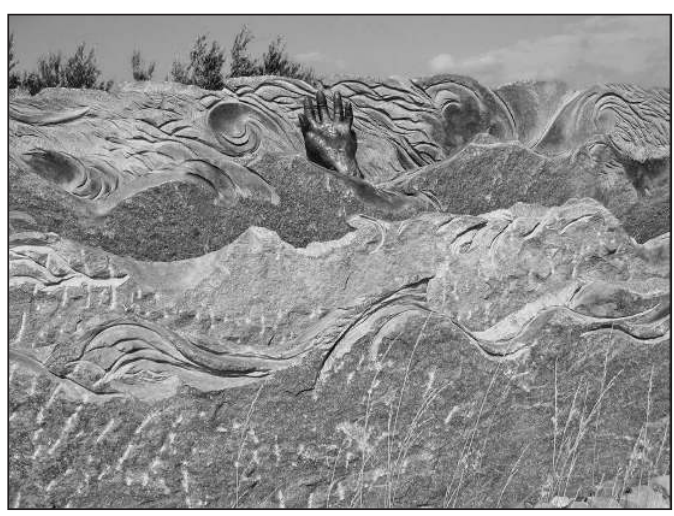


par les opérations de reconstruction ont eu le sentiment d'avoir connu une " guerre », d'avoir vécu dans une région dévastée. Céder devant la mer remettrait en cause ce pour quoi des vies ont été perdues et tant d'efforts fournis. Bien que les Allemands aient connu des épisodes de tempêtes moins dramatiques, la mentalité germanique est également marquée par ces événements. C'est ce qui ressort des contes, légendes et proverbes qui sont rattachés à la mer. Des sacrifices d'humains ou d'animaux, servant à apaiser les esprits en période de tempête ou à assurer une meilleure résistance des digues, ont été une réalité dans ces régions nordiques respectivement jusqu'au $\mathrm{XVI}^{\mathrm{e}}$

Photo 4 : ce monument commémoratif de la tempête de février 1953 (Zélande) se situe à l'emplacement de la dernière fermeture de brêche sur l'île, presque totalement submergée, de Schouwen-Duiveland. Là encore, la force de la mer est symbolisée par le percement apparemment aisé et se répétant, d'un étroit mur de protection érigé par l'homme.

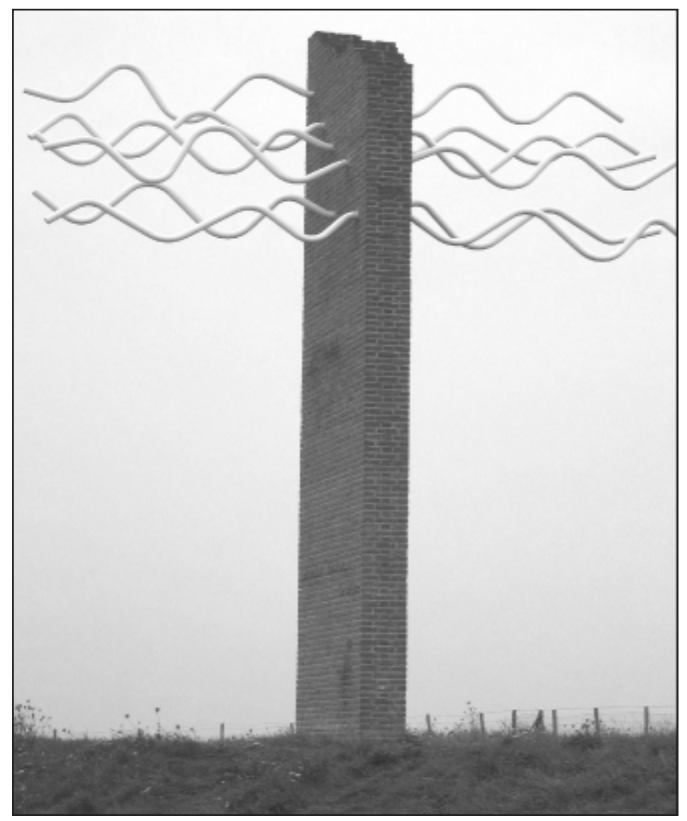

et $\mathrm{XVII}^{\mathrm{e}}$ siècle. Des coutumes aussi excessives ont évidemment un rapport avec une réalité difficile à l'ère médiévale, notamment en Frise du Nord $^{5}$. Plus récemment, la catastrophe de 1962 à Hambourg et dans l'estuaire de l'Elbe et les hauteurs d'eau atteintes en 1976 ont de nouveau marqué les esprits.

Cette importance exacerbée des tempêtes et du risque de submersion marine constitue l'une des explications - si ce n'est l'explication majeure - de l'essor limité de la dépoldérisation dans ces deux pays, ou du moins de dépoldérisations totales par brèches (Bakker et al., 2002). En l'occurrence, les véritables dépoldérisations de polders maritimes restent exceptionnelles aux Pays-Bas ( 1 cas réalisé, 1 cas décidé et des projets uniquement), les autres dépoldérisation sont soient partielles, soit accidentelles, soit totales mais ne concernent alors que des polders d'été ${ }^{6}$ et non de véritables polders maritimes (Goeldner-Gianella, 2008). L'abandon des projets de dépoldérisation de l'Escaut occidental à l'été 2008 est la parfaite illustration de la méfiance néerlandaise à l'égard du retour de la mer (Luteijn, 2008). L'importance sociale des tempêtes pour les Allemands a également limité cette politique. Les dépoldérisations totales qui ont été pratiquées près d'une trentaine de fois en Grande-Bretagne dans des digues principales ne l'ont été que quatre fois en Allemagne, dans l'estuaire de l'Elbe. Globalement dans le pays, cinq autres dépoldérisations totales ont concerné des polders d'été et la grande majorité des dépoldérisations opérées n'a eu qu'un fondement uniquement compensatoire. En Basse-Saxe, il s'agissait de remplacer des marais maritimes perdus du fait de l'implantation de conduites de gaz, de la 
construction du polder du Leybucht en 1991 ou de travaux d'extension portuaire dans la Weser. Ces mesures compensatoires sont également à l'origine de la totalité des dépoldérisations pratiquées à Hambourg, tout autant que des dépoldérisations partielles du Schleswig-Holstein.

\section{L'influence de la participation sociale sur la politique de dépoldérisation}

Sans doute l'implication ou la participation de la société locale favorise-t-elle aussi l'acceptation des dépoldérisations défensives. Cela joue de toute évidence un grand rôle en Grande-Bretagne, où la consultation de la population locale prend différentes formes. Classiquement, l'opérateur d'une dépoldérisation envoie une lettre explicative aux riverains dans un rayon de 10 à $15 \mathrm{~km}$, de même qu'il place des informations dans la presse locale, publie des feuillets d'information et invite les riverains à des journées d'explication ou des réunions publiques, concentrant surtout son action sur des groupes d'intérêt spécifiques ou des voisins inquiets. Ce panel complet a été utilisé à Freiston Shore, où il aurait permis, d'après la RSPB, une grande évolution des regards portés sur le projet de managed realignment (James \& Badley, 2004). Y sont parfois associées des formes d'information plus originales, comme la visite d'autres sites dépoldérisés (utilisée par la RSPB à Nigg Bay), la construction d'un promontoire pour observer les travaux (prévue à Wallasea 2), l'installation d'une caméra durant plusieurs années après la dépoldérisation (Wallasea 1) ou l'utilisation d'experts neutres informer les riverains de l'existence d'un projet (Skinflats dans le Firth of Forth (Midgley \& McGlashan, 2004). Si ces enquêtes ne touchent pas forcément un public très large sur des littoraux reculés, elles éclairent toutefois l'opérateur sur l'avis des riverains. Lorsque cet avis est majoritairement ou trop fortement négatif, il peut renoncer à dépoldériser. Ainsi, les réactions négatives au projet de Weymarks ont conduit le DEFRA à rechercher un site alternatif à ce projet - en l'occurrence Wallasea 1. Ce renoncement est également pratiqué par les ONG, qui ne peuvent se permettre de ternir leur image en imposant un projet impopulaire. Les refus ou les réticences des riverains peuvent tenir à un sentiment d'atteinte à des prérogatives locales : ils peuvent estimer que la rupture des digues entraverait l'accès à la plage (Weymarks, Brandcaster) ou l'utilisation de la digue en tant que sentier public (Weymarks, Brandcaster, Wallasea 1) ; les propriétaires fonciers peuvent critiquer la perte de terres (à Wallasea 1 et 2 à Skinflats) ou jouer de leur influence pour ralentir ou empêcher le processus (Brandcaster, Weymarks). Le fait que les problèmes posés n'ait rien à voir avec des questions de défense contre la mer renforce l'importance de cette consultation, pour faire émerger des enjeux insoupçonnés par les opérateurs de dépoldérisations défensives.

La participation de la société à des projets environnementaux étant incontournable en Europe, depuis la Convention d'Aahrus de 1998, il est évident qu'elle prendra de l'ampleur dans les projets de dépoldérisation. On peut d'ailleurs signaler plusieurs exemples d'implication de la société locale dans des dépoldérisations, bien que celles-ci n'aient pas eu une visée uniquement défensive : c'est le cas dans les projets actuels de dépoldérisation du Perkpolder en Flandre, dans ceux de l'Escaut occidental et dans la baie de la Somme (Goeldner-Gianella, 2008). 
Les dépoldérisations défensives ont-elles un avenir en Europe?

Eu égard à l'élévation du niveau marin et à l'augmentation possible des tempêtes au $\mathrm{XXI}^{\mathrm{e}}$ siècle, il convient de s'interroger sur le devenir de cette politique de dépoldérisation et la diffusion des idées britanniques.

Sur la côte allemande de la mer du Nord, le Plan Général de Protection côtière du SchleswigHolstein a pu paraître assez ouvert en 2001. Il y était dit que : "les retraits ou abandons de digues ne seraient possibles que dans des cas exceptionnels », nécessitant 1) que le niveau de sécurité reste au moins équivalent à l'actuel, y compris dans le cas de l'existence d'une seconde ligne de digues, 2) que la population concernée soit d'accord avec la dépoldérisation envisagée et 3) que les coûts de protection côtière n'augmentent pas pour cette raison (Probst, 1998). Les dépoldérisations n'étaient donc pas officiellement exclues; elles semblaient toutefois irréalisables, ce qu'a confirmé le ministre de l'agriculture du Land la même année. Mais il est vrai que des apports scientifiques nouveaux ont modifié l'idée qu'on se faisait du rôle du schorre dans la protection côtière, et atténué l'intérêt du managed realignment. Niemeyer et Kaiser (2001) ont ainsi montré que la capacité d'atténuation de la houle par le schorre diminuait avec l'augmentation de la hauteur de l'eau durant une tempête, empêchant qu'on tienne compte de la présence d'un schorre pour le calcul de la hauteur des digues. C'est une façon de suggérer que non seulement il est important de continuer à rehausser les digues, mais qu'il ne faudrait pas non plus compter sur un schorre doublé d'une digue-arrière pour assurer une protection efficace de l'arrière-pays, comme dans la technique du managed realignment. Enfin, les dernières réflexions des ingénieurs de la protection côtière vont plus dans le sens d'un triplement de la ligne de digues que de dépoldérisations ! En effet, après une première mesure d'adaptation par rehaussement de la digue de mer, pourraient être construits une nouvelle digue et des tertres artificiels au sein même des polders de première ligne, afin de limiter la vulnérabilité croissante liée à une rupture des première et seconde lignes de digues (Hofstede \& Probst, 2002). Ce serait certes une façon de renoncer, dans le SchleswigHolstein, à l'idée d'une protection totale fournie par la première ligne, mais aussi un signe de retour à la « poldérisation » - quoique plus interne, désormais... En Basse-Saxe, la présence d'une unique ligne de digues limiterait également le recours à la dépoldérisation.

Les Pays-Bas paraissent plus avancés dans leur réflexion. Ils ont lancé en 2002 la campagne «les Pays-Bas vivent avec l'eau » qui les a conduits à prendre davantage la mer en considération : « au lieu de contenir l'eau à nos portes, nous devons lui donner de l'espace et la laisser pénétrer dans nos vies quotidiennes. " (Rijkswaterstaat, mai 2002). Ce retournement de la stratégie traditionnelle de défense contre la mer n'est pourtant pas total, car les aménageurs privilégient un panel de réponses combinant différentes formes d'adaptation, allant de nouvelles avancées sur la mer - par la construction d'îles artificielles au large de la côte - au recul stratégique, en passant par des formes d'adaptation in situ. Il s'agirait par exemple de rehausser le niveau altimétrique des polders en y injectant du sable : les Hollandais, passés maîtres dans le rechargement des plages, 
pourraient aussi recharger des polders grâce aux récents progrès techniques dans l'approvisionnement en sable, le terrassement et la fertilisation des sols. Ainsi, en cas de brèches dans les digues, le niveau des polders ne serait plus inférieur au niveau du plan d'eau marin. De la même façon, on envisage de construire, en certains endroits, des maisons amphibies voire flottantes, pour pouvoir convertir plus aisément certains polders habités en polders de décharge, lors de crues ou de submersions marines (Eudes, 2008). La dépoldérisation pourrait être une troisième technique d'adaptation au changement climatique, mais les projets du Rijkswaterstaat restent indéterminés à ce sujet. Les ingénieurs hollandais ont toutefois été les pilotes du réseau et programme européen ComCoast (2004-2007), portant sur la conception et le test de nouvelles formes de défense contre la mer, telle la dépoldérisation. En dépit de la catastrophe de 1953, ce pays paraît donc assez prêt aux dépoldérisations défensives.

\section{Conclusion}

Les acteurs de la protection côtière (scientifiques, ingénieurs, experts, maîtres d'ouvrage...) jouent un rôle majeur dans l'adaptation au changement climatique et la propagation de nouvelles techniques de défense contre la mer. Mais d'autres acteurs, profitant sans doute des impacts écologiques positifs de la dépoldérisation, cherchent à propager cette forme d'adaptation, qu'ils soient publics, privés ou associatifs. Enfin, les acteurs " passifs » de la société civile jouent également un rôle grandissant dans sa diffusion, tant du fait de l'importance des représentations sociales de la mer ou du changement climatique que de la nécessité d'informer et de faire participer les populations aux projets environnementaux. Les sociétés littorales vont ainsi aider à la propagation ou au refus de techniques novatrices d'adaptation. Mais cette intervention multi-acteurs, qui a commencé à se déployer progressivement sur le littoral, va certainement se muer rapidement en nécessité, pour faire accepter des choix d'aménagement devant désormais traduire une nouvelle philosophie des rivages ${ }^{7}$.

\section{GLOSSAIRE}

Amphibie : capable de vivre dans l'air ou dans l'eau ; conçu pour être utilisé dans l'air ou dans l'eau. Les maisons amphibies évoquées dans le texte peuvent donc être flottantes.

Bassin-versant ou bassin hydrographique : espace géographique alimentant un cours d'eau et drainé par lui. Le bassin a pour limite la ligne de partage des eaux le séparant des bassins hydrographiques adjacents.

Biodiversité : la diversité des formes du vivant. Selon la FAO, la biodiversité comprend la diversité génétique, la diversité spécifique - qui se détermine par le nombre d'espèces, leur rareté, voire leur endémisme - et la diversité écosystémique - qui se détermine par la diversité des milieux dans un espace donné, mais aussi par les interactions entre les espèces qui composent ces milieux. Certaines disciplines s'intéressent aussi à la diversité paysagère, en considérant que chaque écosystème est dépendant des écosystèmes situés dans ses alentours. 
Écosystème : unité structurale élémentaire de la biosphère, composée d'un biotope (espace où sont réunies les conditions physico-chimiques nécessaires à la vie d'une biocénose), associé à sa biocénose (groupement d'organismes).

Élévation eustatique : élévation du niveau de la mer.

Estran : partie du littoral comprise entre les plus hautes mers et les plus basses mers, par conséquent couverte et découverte au rythme des marées. On parle aussi d'étendue intertidale.

Inlandsis : très grand glacier couvrant de vastes étendues terrestres. Les deux inlandsis actuels se trouvent au Groenland et en Antarctique.

Niveau altimétrique : altitude.

Lame de tempête : onde océanique d'origine atmosphérique qui consiste en une surélévation anormale du niveau de la mer au passage d'une dépression atmosphérique vers laquelle convergent les vents soufflant en rafale. L'onde formée est alors poussée vers la côte et amplifiée par les vents.

Oscillation Nord-Atlantique (NAO) : cette oscillation représente les changements typiques de la pression au niveau de la mer dans l'Atlantique Nord, d'un hiver à l'autre. On mesure la différence de pression entre l'anticyclone des Açores et la dépression d'Islande, variable d'une année sur l'autre.

Productivité (organique) : quantité de matière organique produite par les êtres vivants sans intervention humaine, pendant une unité de temps et sur une unité de surface, continentale ou océanique. S'exprime en $\mathrm{t} / \mathrm{ha}$ ou $\mathrm{g} / \mathrm{m}^{2}$, par jour ou par an.

Schorre : terme scientifique d'origine néerlandaise désignant la partie supérieure de l'estran, composée de sédiments fins et couverte d'un tapis de plantes adaptées au sel - les halophytes. Les schorres sont aussi appelés marais salés, et, localement, prés salés en baie du Mont-SaintMichel ou mollières en Picardie.

Subsidence glacio-isostatique : enfoncement progressif d'une surface terrestre en réaction au relèvement progressif et concomittant de terres voisines, occupées durant la dernière glaciation par un inlandsis. Le sud-est de l'Angleterre subit ce phénomène de lent affaissement.

Zone humide : expression désignant tous les milieux aquatiques ou marécageux, en position continentale ou littorale. Les zones humides évoquées dans le texte sont les vasières et les prés salés. 


\section{$R \cdot \dot{E} \cdot F \cdot E \cdot R \cdot E \cdot N \cdot C \cdot E \cdot S$}

J. ALCAMO et al., Europe. Climate Change 2007: impacts, adaptation and vulnerability. Contributions of working group II to the fourth assessment report of the IPCC, in : M.L. Parry, O.F. Canziani, J.P. Palutikof, P.J. Van der Linden, C.E. Hanson (eds), Cambridge University Press, 2007, pp. 541-580.

J.P BAKKER, P. ESSELINK, K.S. DIJKEMA, W.E. VAN DUIN, D.J. DE JONG, «Restoration of salt marshes in the Netherlands », in Hydrobiologia, 2002, n 478, pp. 29-51.

C. CLUS-AUBY, R. PASKOFF, F. Verger, Impact $d u$ changement climatique sur le patrimoine $d u$ Conservatoire du Littoral. Scénarios d'érosion et de submersion à l'horizon 2100. Synthèse, Conservatoire du Littoral, 2005, 44 p.

Commission des Communautés européennes, Livre vert présenté par la commission au conseil, au parlement européen, au comité économique et social européen et au comité des régions : Adaptation au changement climatique en Europe: les possibilités d'action de l'Union européenne, 29.6.2007, $32 \mathrm{p}$.

N.J. COOPER, T. COOPER, F. BURD, «25 years of salt marsh erosion in Essex : implications for coastal defence and nature conservation », in Journal of Coastal Conservation, $\mathrm{n}^{\circ}$ 7, 2001, pp. 31-40.

CWSS, « Coastal Protection and Sea Level rise 2001. Final report», in Wadden Sea Ecosystem, $\mathrm{n}^{\circ} 13,2001,64 \mathrm{p}$.

DEFRA, 2005, Making space for water. Taking forward a new government strategy for flood and coastal erosion risk management in England.

DEFRA (éd.), The UK's fourth national communication under the United Nations framework convention on climate change, 2006, $135 \mathrm{p}$.

DEFRA, Flood and Coastal Defence R\&D Programme, Managed Realignment Review, R\&D Technical Report FD2008, 2002, 212 p.

Department of Environment, Transport and Regions, Potential UK adaptation strategies for climate change, Technical report, 2000.

A.M.C EDWARDS, P.S.J. WINN, The Humber estuary, Eastern England : Strategic Planning of flood defences and habitats", in Marine Pollution Bulletin, $\mathrm{n}^{\circ}$ 53, 2006, pp. 165-174.

EEA (European Environment Agency), Vulnerability and adaptation to climate change in Europe, in EEA Technical Report, ${ }^{\circ}$ 7, 2005, $81 \mathrm{p}$.

EEA (European Environment Agency), Climate change : the cost of inaction and the cost of adaptation, in EEA Technical Report, $\mathrm{n}^{\circ} 13$, 2007, 72 p.

Environment Agency, The state of the environment of England and Wales: coasts, the Stationery Office Ltd., London, 1999, p. 201.

Y. EUDES, «Villes amphibies, îles artificielles », in Le Monde, 03.02.2008.

GIEC Bilan 2007 des changements climatiques. Rapport de Synthèse, Contribution des groupes de travail I, II et III au $4^{\mathrm{e}}$ rapport d'évaluation du GIEC, Genève, 2007, 103 p.

L. GOELDNER-GIANELLA, Dépoldériser en Europe occidentale. Les apports d'une géographie sociale de l'environnement à l'étude du milieu littoral, HDR de Géographie, Université de Nantes, 3 volumes, 2008.

S. GUÉBEN-VENIÈRE, Le rôle des ingénieurs dans la gestion du littoral atlantique français. Le cas de la dépoldérisation en baie de Somme 
et dans le bassin d'Arcachon, Master 2, Univ. Paris 1, 2009, $69 \mathrm{p}$.

J. HANSEN, M. SATO, P. KHARECHA, D. BEERLING, R. ER, V. MASSON-DELMOTTE, M. RAYMO, D.L. ROYER, J.C. ZACHOS, « Target atmospheric $\mathrm{CO}_{2}$ : where should humanity aim ? ", in Open Atmospheric Science Journal, soumis, http://arxiv.org/abs/0804.1126

J. HOFSTEDE, B. PROBST, Integriertes Küstenschutzmanagement in Schleswig-Holstein, 2002, 17 p. (http://www.eucc-d.de/infos/sh kuestenschutz_plan.pdfwww.eucc-d.de/infos/ sh_kuestenschutz_plan.pdf)

L. JAMES, J. BADLEY, Succès d'une dépoldérisation à Freiston Shore (Royaume Uni), in : J .-C. Cornette, P. Triplet, G. Rolland (ed.), Pour une approche intégrée de la protection de la nature en faveur des oiseaux d'eau. L'homme et la nature ont-ils un avenir commun dans les estuaires? Atelier Eurosite/SMACOPI, 22-23/04/2004, 2004, pp. 13-21.

M. LEE, «Coastal defence and the habitat Directive: predictions of habitat change in England and Wales », in The Geographical Journal, $\mathrm{n}^{\circ} 167 / 1,2001$, pp. 39-56.

P. LEROY, « Rendre les Pays-Bas à l'eau ? Le rude défi du changement climatique ", in Septentrion, $\mathrm{n}^{\circ}$ 2, 2006, $7 \mathrm{p}$.

A. LUTEIJN, « Pays-Bas : l'élargissement du chenal de l'Escaut occidental s'accompagnera-til de mesures de restauration de la nature ? », in Escaut sans Frontières Info, 2008, pp. 43-44.

MEDD, Quatrième communication nationale à la Convention cadre des Nations unies sur les changements climatiques, 2006, 232 p.

L.B. MYATT, M.D. SCRIMSHAW, J.N. LESTER, «Public Perceptions and Attitudes towards a Current Managed Realignment Scheme:
Brancaster West Marsh, North Norfolk, UK», in Journal of Coastal Research, $n^{\circ} 19 / 2,2003 a$, pp. 278-286.

L.B. MYATT, M.D. SCRIMSHAW, J.N. LESTER, «Public perceptions and attitudes towards a forthcoming managed realignment scheme: Freiston Shore, Lincolnshire, UK », in Ocean \& Coastal Management, $\mathrm{n}^{\circ} 46,2003 \mathrm{~b}$, pp. 565-582.

L.B. MYATT, M.D. SCRIMSHAW, J.N. LESTER, «Public perceptions and attitudes towards an established managed realignment scheme: Orplands, Essex, UK », in Journal of Environmental Management, $\mathrm{n}^{\circ}$ 68/2, 2003c, pp. 173-181.

S. MIDGLEY, D.J. Mc GLASHAN, « Planning and management of a proposed managed realignment project : Bothkennar, Forth estuary, Scotland », in Marine Policy, $\mathrm{n}^{\circ}$ 28, 2004, pp. 429-435.

A. MIOSSEC, La gestion de la nature littorale en France Atlantique. Étude comparative (RoyaumeUni, Pays-Bas, Espagne, États-Unis), Doctorat d'État, UBO, vol. 1, 1993, 469 p.

H. D. NIEMEYER, R. KAISER, « Hydrodynamische Wirksamkeit von Lahunugen, Hellern und Sommerdeichen », in Die Küste, ${ }^{\circ}$ 64, 2001, pp. 15-60.

ONERC, Stratégie nationale d'adaptation au changement climatique, Paris, La Documentation française, 2007, 97 p.

R. PASKOFF, Potential implications of sea-level rise for France », in Journal of Coastal Research, $\mathrm{n}^{\circ} 2$, 2004, pp. 424-434.

W.T. PFEFFER, J.T. HARPER, S. O'NEEL, « Kinematic Constraints on Glacier Contributions to 21 st-Century Sea-Level Rise », in Science, ${ }^{\circ}$ 321/5894, 2008, pp. 1340-1343. 
B. PROBST, "Leitbild und Ziele des Küstenschutzes in Schleswig-Holstein », in Wasser \& Boden, $\mathrm{n}^{\circ}$ 50, 1998, pp. 19-24.

S. RUPP-ARMSTRONG, R. NICHOLLS, « Coastal and Estuarine Retreat: A Comparison of the Application of Managed Realignment in England and Germany », in Journal of Coastal Research, $\mathrm{n}^{\circ}$ 23/6, 2007, pp. 1418-1430.

N. SCHOENENWALD, « L'Europe du NordOuest sur le rail des tempêtes », in M. Tabeaud (dir.), Ile-de-France, avis de tempête force 12, Paris, Publications de la Sorbonne, 2003, pp. 41-55.

P. WAGRET, 1959, Les polders, Paris, Dunod.
$\mathrm{N} \cdot \mathrm{O} \cdot \mathrm{T} \cdot \mathrm{E} \cdot \mathrm{S}$

1. (...) qui représentent un cinquième de toutes les formes de dépoldérisations réalisées ou projetées jusqu'en 2008 en Europe.

2. Il possède 4000 ha de marais endigués et doit encore en acquérir 27000 ha.

3. Selon les sources, les surfaces submergées s'étendaient sur 38000 ha en Allemagne, 60000 à 100000 ha dans le sud-est de l'Angleterre et 200000 ha environ dans le sud-ouest des Pays-Bas. En plus de pertes animales, 47000 bâtiments ont été endommagés en Zélande, de même que 24000 en Angleterre et plusieurs milliers en Allemagne. WAGRET, 1959 ; IDG, 1993 ; et http://www.metoffice.gov.uk/corporate/ pressoffice/anniversary/floods1953.html

4. Littéralement « je lutte (contre la mer) et j'émerge $»$.

5. En Frise du Nord, on fait souvent référence au village de Rungholt, disparu durant la tempête de 1362. 10000 habitants de l'Eiderstedt et des Dithmarschen auraient été victimes de la tempête de 1216 et 6000 de celle de 1634 (Wagret, 1959).

6. Un polder d'été est un polder pourvu d'une digue très basse (2 à $3 \mathrm{~m}$ ), qui laisse passer les plus hautes eaux. Il n'a pas de vocation défensive car il est situé juste au-devant et au contact des véritables polders, qui sont eux pourvus de digues protectrices très hautes. Rendre ces polders d'été à la mer n'a pratiquement aucune incidence en termes défensifs.

7. Nous empruntons cette dernière expression, bien connue des géographes littoralistes, à André Guilcher. 
$R \cdot E \cdot S \cdot U \cdot M \cdot E ́$

Les acteurs publics de la protection côtière jouent un rôle majeur dans l'adaptation au changement climatique en Europe du nord-ouest et dans la propagation de nouvelles techniques de défense contre la mer, comme la dépoldérisation. Mais d'autres acteurs, davantage tournés vers la protection de la nature, cherchent eux aussi à promouvoir cette technique, qu'ils soient publics, privés ou associatifs. Dans le cas particulier d'une adaptation par la dépoldérisation, c'est une gestion multi-acteurs qui tend à se mettre en place, de même qu'une participation croissante de la société à l'échelle locale.

\section{Summary :}

In north-western Europe, public coastal defence players play a major role in the adaptation to climate change and the propagation of new sea defence techniques, as de-polderisation. Other actors more interested in nature conservation also try to promote this technique, whether they belong to the public, the private or the community world. In the specific case of adaptation through de-polderisation, a multipartners management is being implemented, as well as the increasing participation of society on the local scale. 